\title{
Diamond coated artificial cardiovascular devices
}

\author{
Hongjun Zeng ${ }^{1}$, Robert Jarvik ${ }^{2}$, Grace Catausan ${ }^{l}$, Nicolaie Moldovan ${ }^{l}$, and John Carlisle ${ }^{l}$ \\ ${ }^{1}$ Advanced Diamond Technologies, Inc. 48 E Belmont Drive, Romeoville, IL 60446, USA \\ ${ }^{2}$ Jarvik Heart, Inc., 333 West 52nd Street, New York, NY 10019. \\ zeng@thindiamond.com
}

\begin{abstract}
Ultrananocrystalline diamond (UNCD), an extremely smooth, low cost diamond coating was successfully developed herein for antithrombogenic application which requires high biocompatibility, low wear, low friction, and chemical inertness. The substrate materials utilized in the Jarvik 2000 ventricular assist device (VAD), silicon carbide and titanium alloy, provide an excellent substrate match for UNCD integration. The paper addresses the development of medical-quality UNCD films to significantly improve the knowledge base regarding the defect mechanisms of UNCD films, to reduce or eliminate known wear-inducing imperfections in the film, and to thoroughly characterize and test the films as well as assembled UNCD-coated VADs. After the defect reduction and seeding experiments to improve film adhesion and coating quality, the best candidate deposition method has been downselected for coating and assembly of VAD parts from Jarvik Heart. The coated and assembled devices have been tested with mechanical and blood-simulating fluid hydrodynamic testing at Jarvik Heart for full verification of the new coating technology. UNCD interface takes advantage of combining unmatched durability and antithrombogenicity.
\end{abstract}

\section{Introduction}

In the US only, more than 5 million people are impacted by and more than 280,000 deaths are caused by heart failure each year. [1] The annual treatment cost is estimated at between $\$ 10$ and $\$ 40$ billion. Behind these sobering numbers, medical therapies implanted cardiac devices and surgical techniques to fight this scourge are realizing significant improvements. Among the most significant of these efforts, 
ventricular assist devices (VADs) maintain the quality of life for patients awaiting transplant or they can provide a period of time for recovery or healing of the patient's own heart. Mechanical heart valves (MHVs) are even more widely used to replace failed heart valves. Both of these artificial cardiovascular devices (ACDs) have saved millions of lives since 1970s. However, ACDs still exhibit a number of drawbacks among which thromboembolism is the most serious. Anticoagulant treatment effectively treats the risk of thrombosis for the majority of patients who receive these implants, however, maintaining consistent long-term concentrations of anticoagulants is challenging. On other hand, mechanical durability of the long-term or even permanent implantable devices, especially for their moving components still needs to be further improved. For example, mechanical heart valves made of pyrolytic carbon wear at the hinge area, release debris inside the body and cause osteolysis [2]. A better material with both good biocompatibility and wear resistance, is as crucial as a better design for next generation of ACDs [3].

So far it has not been successful to find a "perfect" material that can meet both biocompatibility condition to drop anticoagulant usage and durability condition to avoid the mechanical failure. However, it is practical to coat a thin film to convert the current structural material to be more biocompatible and durable. Previous work on implantable devices with surface coatings of diamond-like carbon (DLC) [4] has demonstrated reduced interactivity between the coating and human blood clotting factors such as the protein fibrinogen [5-6]. However, high mechanical stress levels were witnessed during the integration of DLC with the structural materials. Crevice corrosion in the structural materials through the small crack of the DLC film was reported when the coating was applied on hip joints and metatarsophalangeal joints. [7-8] Diamond, with its extreme chemical inertness, hardness, and low level of interaction with the immune system [9], is an ideal material to eliminate thrombus originating on the VAD, but typical diamond films are too rough $(0.1-1.0 \mu \mathrm{m}$ RMS $)$ due to their growth chemistry. The UNCD films proposed for this work consist of 2-5 nm grains of crystalline diamond separated by atomically-abrupt 
grain boundaries that contain a mixture of $\mathrm{sp}^{3}$ and $\mathrm{sp}^{2}$ carbon. The use of UNCD, can lower surface roughness by up to three orders of magnitude as compared to conventional microcrystalline diamond, and with its overall bio-inertness, extreme resistance to wear, and very low friction coefficients, should significantly improve upon the advantages previously demonstrated with other diamond films [10-11].

In this report, we choose to coat a Jarvik 2000 Heart [12], a lifetime ventricular assist device as a good example to demonstrate UNCD integration with cardiovascular devices with complicated 3diamensional shapes. Under the current situation that heart recovery time or the waiting time for a heart transplant is extremely long (the ratio of patients on waiting lists to donor hearts is about $75: 1$ ), such that a temporary or even a permanent artificial heart is essential for the patients. The core components of a Jarvik 2000 Heart [13] is made of silicon carbide (SiC) and Ti alloy (Ti-6Al-4V). These two materials take advantage of high mechanical durability, ease of shaping, relatively low cost and decent biocompatibility, however they are not as biocompatible as pyrolytic carbon (PyC). On the other hand, PyC is not mechanically durable under high-speed dynamic conditions. Our recent work has demonstrated that UNCD can be as biocompatible as $\mathrm{PyC}$, if not more, and significantly more biocompatible than SiC. [14] Due to its unmatched smoothness, UNCD could be an ideal choice to eliminate friction on the interface between bearings of the VADs. This paper presents a robust and uniform UNCD integration on complicated 3dimensional components of the VADs.

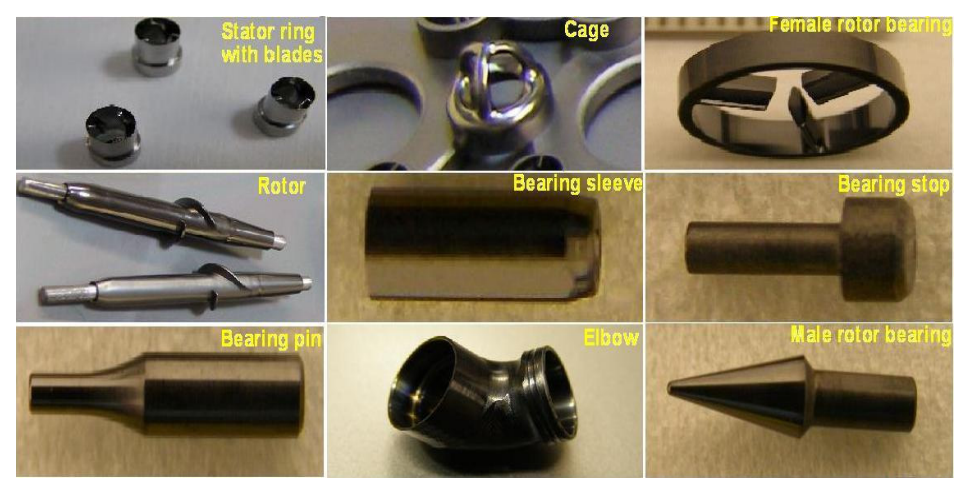

Fig. 1 Components of Jarvik Heart

\section{Experiment}

The UNCD coating was conducted in a tungsten hot-filament chemical vapor deposition (HFCVD) reactor. Diamond film growth starts with substrate seeding using commonly-accepted diamond seeding 
processes with slurries containing suspended diamond nanoparticles [15]. As a result, a high density of diamond particles (usually $10^{9}-10^{12} / \mathrm{cm}^{2}$ ) on the substrate ensures appropriate seeding density which accelerates the onset of diamond film deposition and provides for a more uniform growth rate and thickness. The reactor setup and seeding processes are similar to those reported previously [16]. High purity UNCD deposition uses an $\mathrm{H}$-rich gas system with a $\mathrm{CH} 4 / \mathrm{H} 2$ ratio as high as $10 \%$ employed, which was studied in a recent report [17]. The UNCD film thickness practiced for these parts is in the range of $1.5-2.5 \mu \mathrm{m}$.

The components to be coated were provide by Jarvik Heart, Inc., involving two basic structural materials: $\mathrm{SiC}$ and Ti alloy (Ti-6Al$4 \mathrm{~V})$. The special task in this work was coating UNCD on 3-dimensional components of the VADs with different shapes as shown Fig. 1, some of them needing a coating on their inner surfaces. First, to ensure a uniform coating, a uniform seeding process is required. Because the diamond seeds are weakly attached to the substrate surface via a Van der Waals Force,
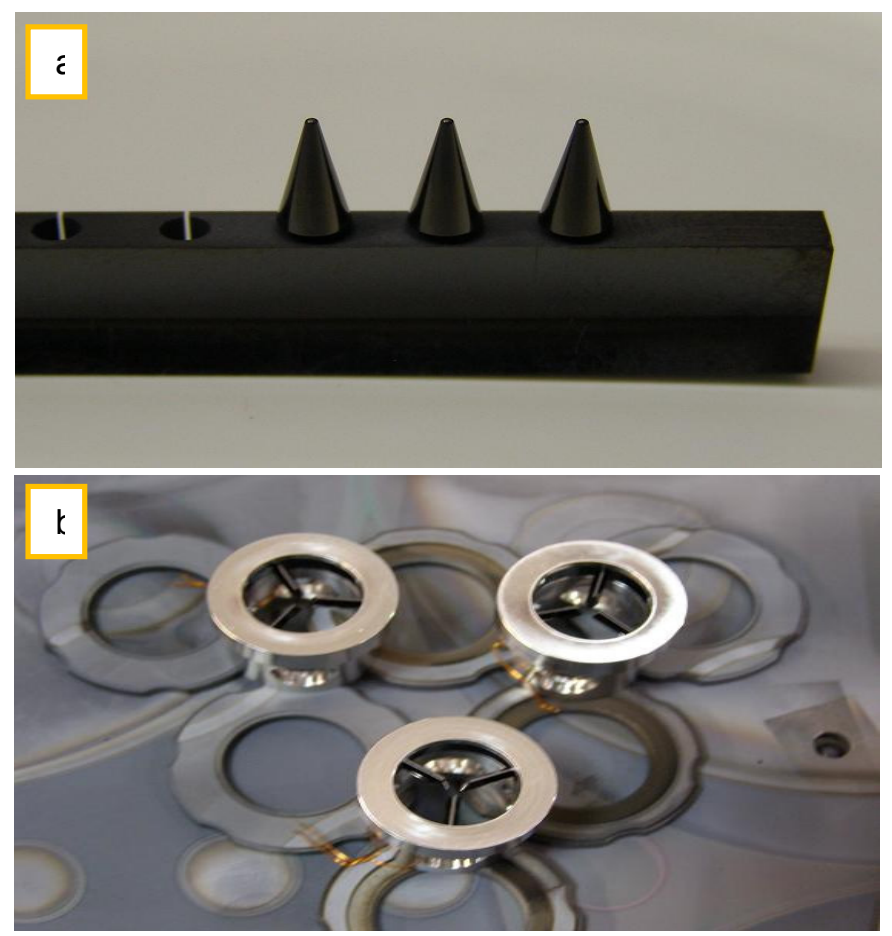

Fig. 2 Fixtures customized to a) handle the male rotor bearings during and after seeding process, ensuring that the cone faces to be coated by diamond, while avoiding diamond/graphite deposition on the handle part, and b) handle the female rotor bearings during deposition, to ensure only the central tongues and beams receive diamond, while the ring of the wheel is not coated with diamond.

the attachment of the seeds can be easily disrupted by other forces provided by objects that touch the seeds. Therefore, fixtures were customized for handling different components. Fig. 2a shows an example for handling a male rotor bearing as an example. These fixtures only touch spots on the part surface where diamond deposition is not required. Second, the hollow components in need of a UNCD coating on their inner surfaces were placed on customized loading fixtures able to hold them in specific angles, so that the gas and diamond precursors can pass and react on the inside surface without turbulence. Fig. $2 \mathrm{~b}$ shows an 
example of handling a female rotor bearing. It is convenient that a specific fixture can combine functions of handling, seeding and coating a specific component. These fixtures only touch spots on the parts' surface where diamond deposition is not critical. Processing operators conducting the deposition always hold the parts to be coated by the fixture, without touching any portion of the parts themselves. The fixture material should not be a source of contamination for the diamond deposition, should not be dissolved by the seeding slurry, and should be strong enough to survive ultrasound, hot gas chemistry and thermal cycling during the diamond deposition. Therefore, high density graphite, silicon carbide, and refractory metals were chosen as fixture materials. Finally, as compared with the standard thin planar geometry of most semiconductor industry wafer products, complex 3D parts for VADs have a sizable height. When they are coated, the deposition rate changes as a function of height. After setup upgrading, the control of the UNCD film thickness was possible in a range from $1.6 \mu \mathrm{m}$ to $2.4 \mu \mathrm{m}$ within entire of the height of all components (maximum height up to $20 \mathrm{~mm}$ ).

In the evaluations on the film, a high resolution SEM was used to study the UNCD grains and surface morphology. The accelerating voltage, aperture size and working distance of the SEM were $10 \mathrm{kV}, 10 \mu \mathrm{m}$ and $10 \mathrm{~mm}$, respectively. Atomic Force Microscopy (AFM, Veeco Nanoscope IIIa) was employed to measure the surface roughness of the samples before and after diamond deposition. AFM tapping mode was used to scan a $5 \mu \mathrm{m} \times 5 \mu \mathrm{m}$ area to extract the root mean square (RMS) roughness. Raman spectra were obtained using a spectrometer (Control Development 2DMPP) with an frequency-doubled neodymium-doped yttrium aluminum garnet (Nd:YAG) laser. The laser wavelength and power were 532 $\mathrm{nm}$ and $5 \mathrm{~mW}$, respectively. Film stress was evaluated by a Flexus Stress Measure System (Tencor FLX2320), with input of thicknesses of the diamond film and the parameters of the substrate. The film adhesion was characterized via a Nanovea Scratch Test station and a Rockwell Ball Indenter with a Thomson Precision Ball (ceramic, 1/16"). The test follows the ASTM C1624-05, i.e. "Standard Test Method for Adhesion Strength and Mechanical Failure Modes of Ceramic Coatings by Quantitative 
Single Point Scratch Testing". Contact angle measurement was conducted with micro-volume pipettes which could dispense deionized-water droplets with a diameter less than $100 \mu \mathrm{m}$ to avoid impact of the gravity. A high resolution camera was used to capture side-view images of droplets 5 seconds after their dispensing, when the droplets become stable but do not significantly evaporate. Contact angle measurement was conducted on the images with an angle measurement software (Scion Image Beta 4.0.2). The characterized components were sent to Jarvik Heart and were inspected for any coating defects or geometry change after coating. The qualified parts were then assembled into Jarvik 2000 ventricular assist devices, followed by in vitro plasma circulating evaluation. In an in vitro test, artificial plasma was circulated through the devices for a few weeks. Post-test evaluations including SEM, profilometry, Raman and contact angle were performed to investigate if the diamond coating was changed in morphology, roughness and diamond chemistry as compared with data acquired before the experiments
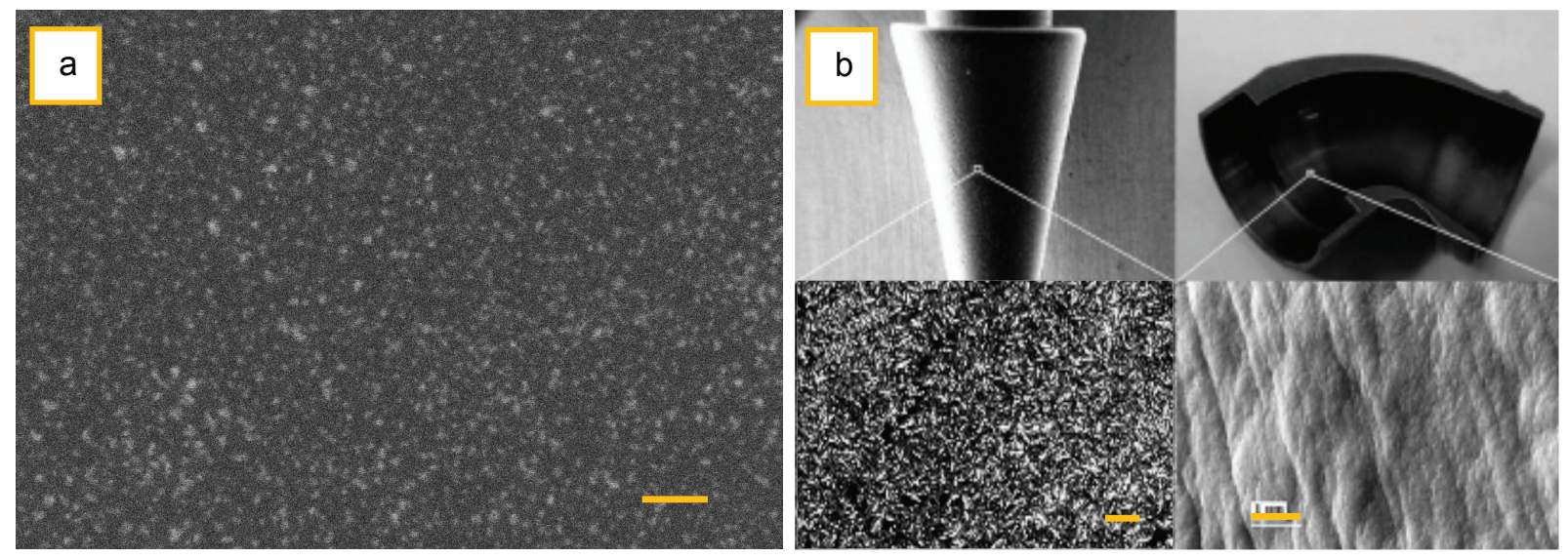

Fig 3 a) SEM image of diamond seeds distribution on a SiC substrate surface, b) SEM image of UNCD on surface of a rotor bearing (left) and on inner surface of an elbow-shape tube (right). Scale bar in Fig. $3 \mathrm{a}$ is 100 $\mathrm{nm}$ and scale bars in Fig. $3 \mathrm{~b}$ are $1 \mu \mathrm{m}$.

at Jarvik Heart.

\section{Results}

With carefully designed seeding and handling fixtures, the seeds density on the substrates (both SiC and Ti alloy) after the seeding process is as high as reported, in the order of $10^{12} / \mathrm{cm}^{2}$. In addition, as SEM image shows in Fig. 3a, the seeds distribution is very uniform (Fig. 3a), with 5-10 nm single diamond particle size and 10-15 $\mathrm{nm}$ particle-particle distance in average. Such a high uniformity of seeds 
distribution eliminates the asperities caused by any congregation of the multiple seeds and pin-holes (discontinuities) in film caused by any locally seeds absence. Under high pressure and high-speed movement, it is very important to have minimal asperities and pin-holes densities, which otherwise would increase friction or the possibility of scratching the matching surface. Fig. $3 \mathrm{~b}$ shows a male rotor bearing made of SiC and an L-shape tube made of TiVAl, coated with UNCD on the cone tip and inside of the tube, respectively. The inner surface was measured after the tube was cut by electrical discharge machining (EDM) after UNCD coating. Their corresponding SEM images show that all targeting areas are covered by UNCD with typical UNCD Raman signature as shown in Fig. 7c, with peak components at $1170 \mathrm{~cm}^{-1}, 1350 \mathrm{~cm}^{-1}, 1470 \mathrm{~cm}^{-1}$, and $1560 \mathrm{~cm}^{-1}$. While the peaks at $1350 \mathrm{~cm}^{-1}$ and $1560 \mathrm{~cm}^{-1}$ represent so-called D-bond (disordered carbon) and G-bond (crystallized graphite), peaks at $1170 \mathrm{~cm}^{-1}$ and 1470 $\mathrm{cm}^{-1}$ represent transpolyacetylene, specifically dwelling in grain boundaries of NCD or UNCD [18]. As has been reported, UNCD with such a Raman signature has very high, up to 97\% sp3 purity. [17]. 


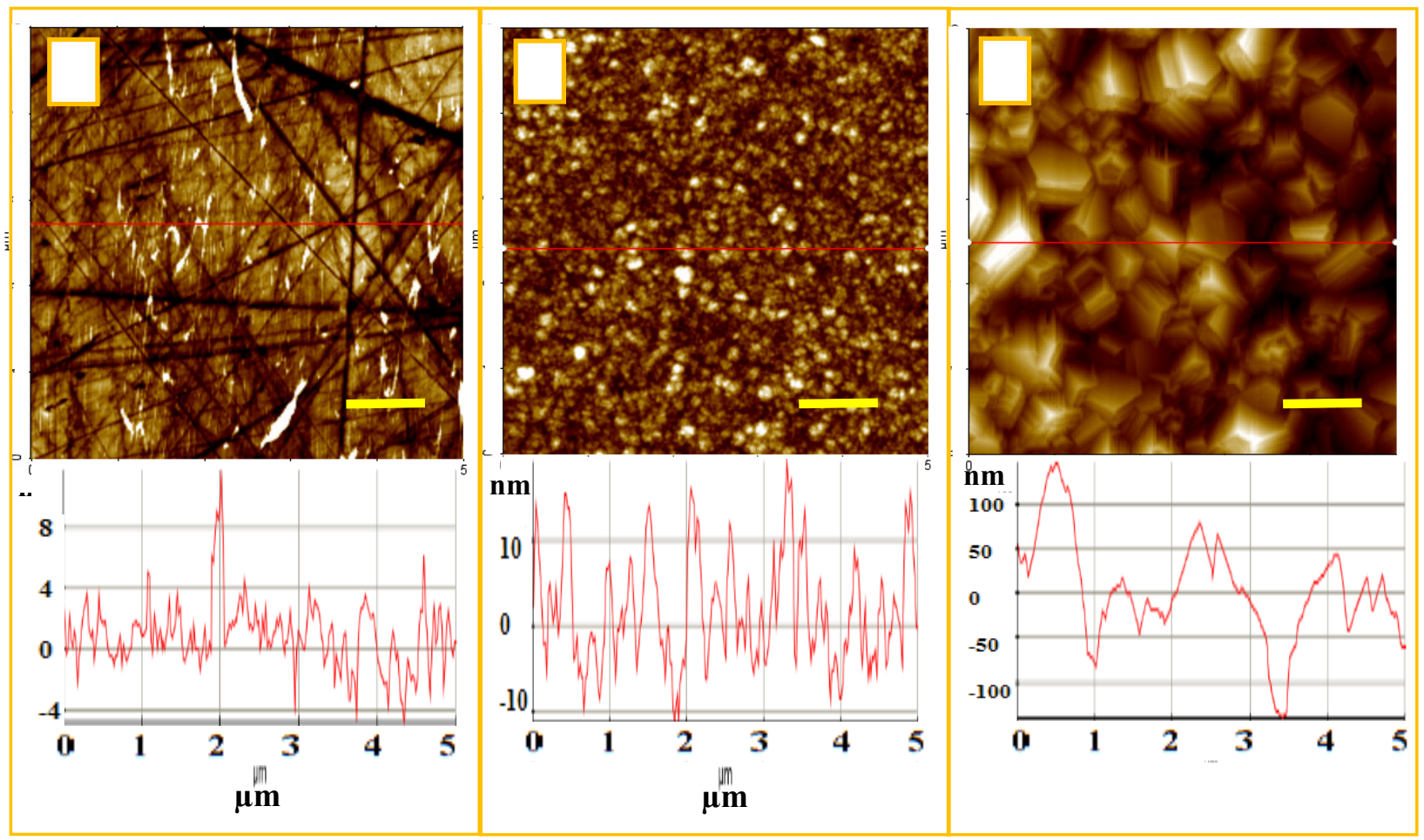

Fig.4 AFM images (top) and one-dimensional morphology (bottom) of a) a fine-polished SiC coupon, b) UNCD deposited on the $\mathrm{SiC}$ coupon and c) conventional nanocrystalline diamond deposited on $\mathrm{SiC}$ coupon. Lateral scan range $=5 \mu \mathrm{m}$ and vertical scan range is with a unit of $\mathrm{nm}$. Scale bar of all images represents $1 \mu \mathrm{m}$. 
The surface roughness of the UNCD coating was investigated with AFM. Fig. 4a and 4b show a surface morphology of a fine polished $\mathrm{SiC}$ coupon before and after the UNCD deposition. Although their topview morphologies look different, the UNCD coating does not bring significant change of surface roughness. The root mean square roughness of the uncoated and coated surface was measured as 3-6 nm and 5-8 nm respectively, in a $5 \mu \mathrm{m} \times 5 \mu \mathrm{m}$ scan range. In contrast, roughness of a typical conventional nanocrystalline diamond surface (Fig.4c) can easily reach $>100 \mathrm{~nm}$ when thickness goes higher than 1 $\mu \mathrm{m}$, which could bring about larger friction.

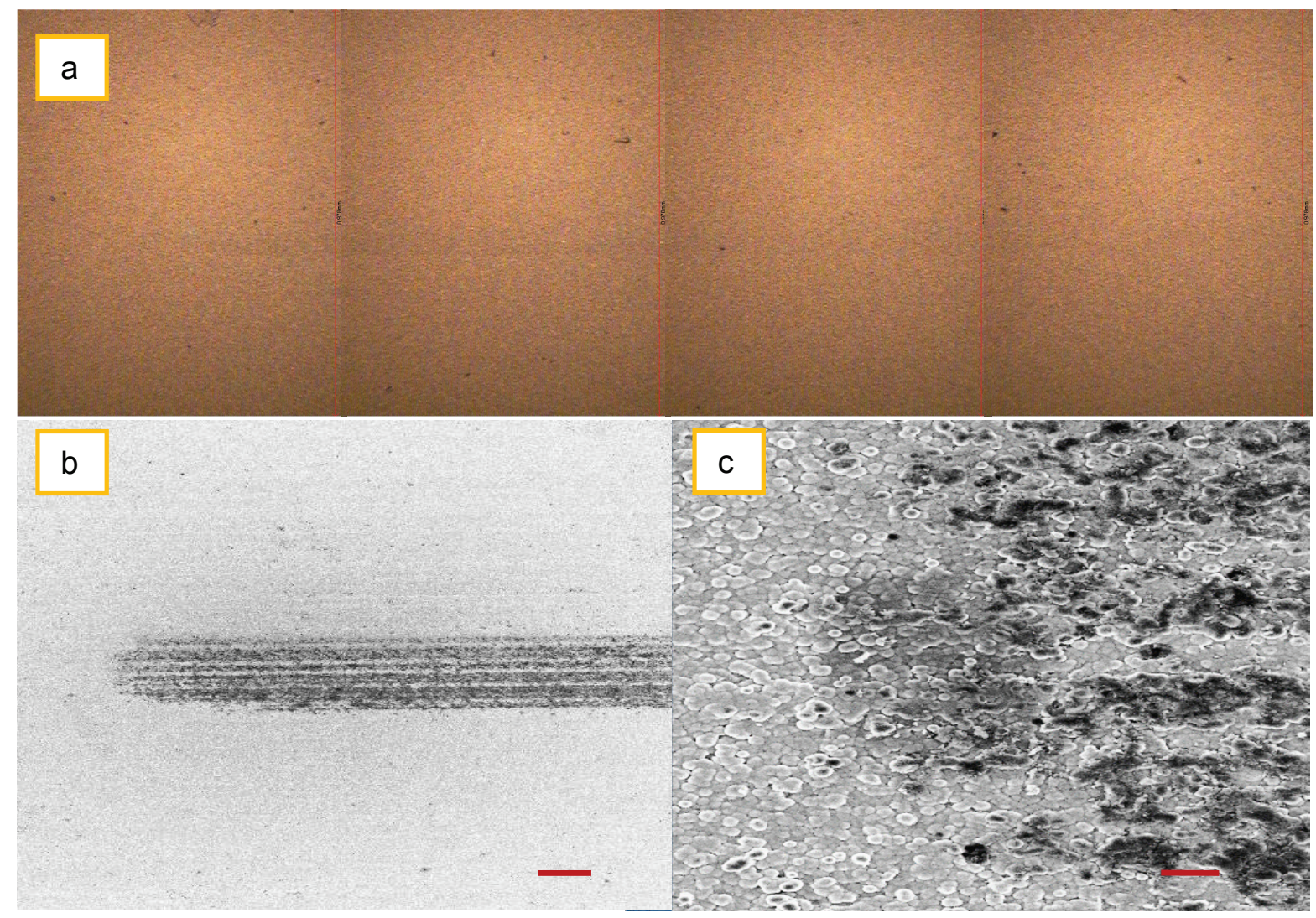

Fig.5 Scratch test on UNCD film coated on a rough SiC substrate. a) a very shallow trace shown under optical microscope after the test, combined by 4 micrographs; b) SEM image of the trace end at 200-N load and c) magnified SEM image of the trace end. Scale bars in b) and c) represent $100 \mu \mathrm{m}$ and $10 \mu \mathrm{m}$ respectively.

For moving components such as leaflets of mechanical heart valves and bearings of heart pumps reported in this paper, failure of devices are mainly due to surface instability and accumulation of 
deposits affecting the surface tribology and leading to wear through attrition of particles from the rubbing surfaces. Such failure causes in long term functioning devices are well-known in the seals and bearings industry. While DLC is one of the commonly reported surface coatings to address these issues, UNCD with characteristics measured above, seems to be even more promising. We measured a $>30 \mathrm{~nm}$ RMS (root mean square) roughness on a $2-\mu \mathrm{m}$-thick commercially available DLC film, while roughness measurements on UNCD yielded values about 50\% less than for DLC at the same film thickness. Evidently, a smoother surface provides not only lower friction which leads to lower wear, but also lower protein/cell attachment rate. Lower protein/cell attachment is related to lower thrombus formation. In addition, our previous studies show that hydrophobic surfaces (with large contact angle) have generally better biocompatibility than hydrophilic surfaces [5]. While contact angles of DLC were measured in the range of $50-60^{\circ}$, UNCD's contact angle is usually $>80^{\circ}$, which suggests a basis for an even better biocompatibility.

An aggressive scratch test was conducted on the UNCD-coated SiC coupons. Within $4 \mathrm{~mm}$, weight load is linearly applied on the sample through a $1 \mathrm{~mm}$ radius ceramic ball from $0.2 \mathrm{~N}$ to $200 \mathrm{~N}$. Due to very strong adhesion, no film crack, wrinkle, chipping or delamination were witnessed. When the test was conducted on the UNCD-SiC sample with roughness $<10 \mathrm{~nm}$ as mentioned above, no scratch trace at all was noticeable under the microscope. When the test was conducted on a rougher coating (RMS $=50 \mathrm{~nm}$, roughness modified by roughening a carbide substrate but with same UNCD coating), a very shallow scratch trace was observed, as shown in Fig.5a. SEM images in Fig. 5b-c reveal that the trace is actually composed of debris left from the ceramic ball scraped off by the hard and protruded structures of the UNCD-SiC sample. This was also observed in previous work with UNCD-coated pyrolytic carbon leaflets for mechanical heart valves. [14] The contact area between the ceramic ball of the scratch tester and the UNCD surface is circular with a diameter of approximately $200 \mu \mathrm{m}$ at the end of the test, therefore, with a load of $200 \mathrm{~N}$, one can calculate that the maximum pressure applied on the film was 
about $6 \times 10^{9} \mathrm{~Pa}$. The UNCD-SiC integration has a measured bond strength between that of the UNCD and silicon carbide $(\mathrm{SiC})$ exceeding the compressive strength of the $\mathrm{SiC}$ itself. While absolute failure-free run times in real in vivo environment depend on a given set of conditions, the data shown are for severe test conditions. Under these severe conditions, UNCD-coated components tested in validation trials have shown up to $20 \times$ longer lifetimes than uncoated $\mathrm{SiC}$ counterparts.

The extremely strong adhesion between the underlying $\mathrm{SiC}$ and the covering UNCD is mainly determined by the potential of forming gradual transitions from carbide bonds to diamond bonds between the two materials. Optimized deposition recipes for interfacial shear stress reduction also reduce the likelihood of mechanical failures and delamination. The developed pre-deposition surface treatment helps in forming covalent bonds between $\mathrm{SiC}$ and diamond, without requiring any extra adhesion layer. While a huge compressive stress, higher than $800 \mathrm{MPa}$ was measured on DLC films on Si wafers, in asdeposited UNCD film on Si, stress is usually less compressive, usually less than $300 \mathrm{MPa}$. Annealing DLC films can only drop the stress to about $700 \mathrm{MPa}$ compressive, while UNCD film's stress can be decreased to a value as low as $\pm 100 \mathrm{MPa}$ ( + and - represents tensile and compressive) with a newly developed stress releasing method. [14] A high film stress not only leads to a weak film adhesion but also causes a mechanical distortion of the coated components. Moreover, to improve adhesion, DLC coating usually employs an adhesion layer. Once the delamination occurs, the adhesion layer with lower biocompatibility gets exposed to the tissue and blood environment, potentially causing thromboembolic complications. [7-8]

Corrosion-related delamination is of practical concern for implantable applications. Such a failure mode can be caused by 1) pin-holes and cracks in the top film and 2) reaction of the structure material with the environmental fluids. To prevent pin-holes and cracks in the film, we have provided a suited UNCD coating strategy, i.e., enhance nucleation at the beginning of UNCD deposition, and used UNCD thickness to exceed the pinhole-free threshold. Minimizing film cracking is realized by minimizing film 
stress as discussed above and enhancing film adhesion. At a hard-hard contact interface, a smooth finish of the film lowers the friction and interfacial shearing forces, which also lowers risk of film cracking. In the last several years the authors group has significantly developed the film adhesion by means of surface pretreatment and stress control. Actually we discovered that for a SiC-SiC contact, even if only one side of $\mathrm{SiC}$ is coated with $\mathrm{UNCD}$, due to the huge friction decrease, the surface wear and particle release can be dramatically lowered in the contact area, which will be further detailed in a future publication. Even in case of any pinhole or small delamination, the exposed SiC, as a FDA accepted material that is much more chemically stable and mechanically durable than $\mathrm{Si}$, can still be much more resistant to the crevice corrosion.
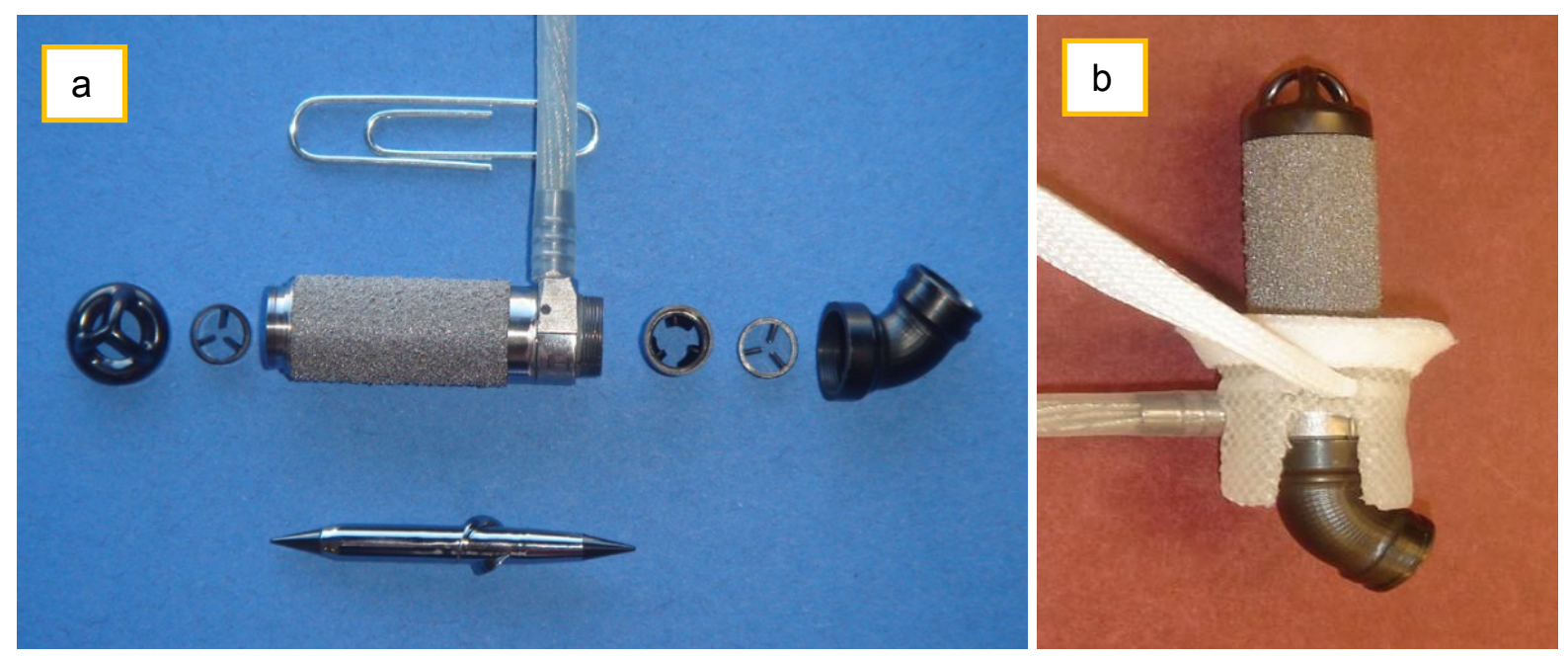

Fig. 6 a) UNCD coated parts were used for all surfaces except the rotor and bore of the housing and b) assembled UNCD coated Jarvik 2000 Heart pump with apical sewing cuff.

Apart from the characterization above, thrombin generation of fresh human platelets on UNCD was intensively studied in another report. [14] The thrombin generation on UNCD was statistically as low as that generated from the platelets without exposing to any foreign substance, equal or lower than commercially available $\mathrm{PyC}$, and significantly lower than that of SiC and NCD/MCD with grain size larger than UNCD [19]. The UNCD-coated parts were assembled into a Jarvik 2000 Heart (Fig 6a and 6b). As an in vitro test, artificial plasma was circulated through the device for 3 weeks, with the rotor 
speed up to 35,000 RPM and plasma flow rate up to $3.5 \mathrm{~L} / \mathrm{min}$. After the test, the device was dissembled and evaluated. No delamination and physical defects were witnessed with neither visual, nor optical microscopy and SEM inspections. We continuously scanned the whole surface of the coated parts under SEM with a secondary electron detector with high contrast imaging between UNCD and SiC. The SEM scan did not capture any pieces of delamination or loss. The male/female bearings and the elbow-shape tube (EDM cut into half), which were the mostly stressed parts due to high-speed rotation of the rotor and backpressure, were carefully inspected under SEM. As shown in Fig. 7a-b. No significant UNCD texture change was witnessed before and after the test. Raman spectra on the UNCD films coated on these parts show that diamond signature before and after the test was not changed. (Fig. 7c). As we know from previous work, change of surface chemistry of a diamond film could be simply reflected by contact angle change. We have studied that an as-deposited UNCD surface is hydrophobic to a deionized water droplet due to the film's concentrated hydrogen surface termination. [17] If the surface is degraded by oxidation or plasma deposit during the test, the contact angle would become dramatically smaller. However, we measured the contact angle changes before and after the test smaller than the measuring errors, i.e., $\pm 5^{\circ}$ around $90^{\circ}$ (insert in Fig. $7 \mathrm{c}$ ), demonstrating that almost no chemical property changed during the test occurred. The test result is very encouraging, which triggers more experiments such as even longer time test, post plasma analysis, whole blood tests and eventually a systematic in vivo animal study in the near future.

\section{Conclusion}

Durable and biocompatible UNCD has been integrated onto components of a Jarvik 2000 Heart. The seeding process, film stress control, and 3-dimensional parts coating have been developed to enhance the adhesion of UNCD on the components and to provide uniform coatings. SEM, AFM and Raman measurements revealed a high purity UNCD deposition. The aggressive scratch test and plasma circulation test show that the film integration is very robust. The positive results of these experiments, 
as well as a successful 3-week artificial plasma test, open a gate for more research on diamond coated cardiovascular devices such as in vivo tests and enable the perspective of more reliable and safe cardiovascular devices.

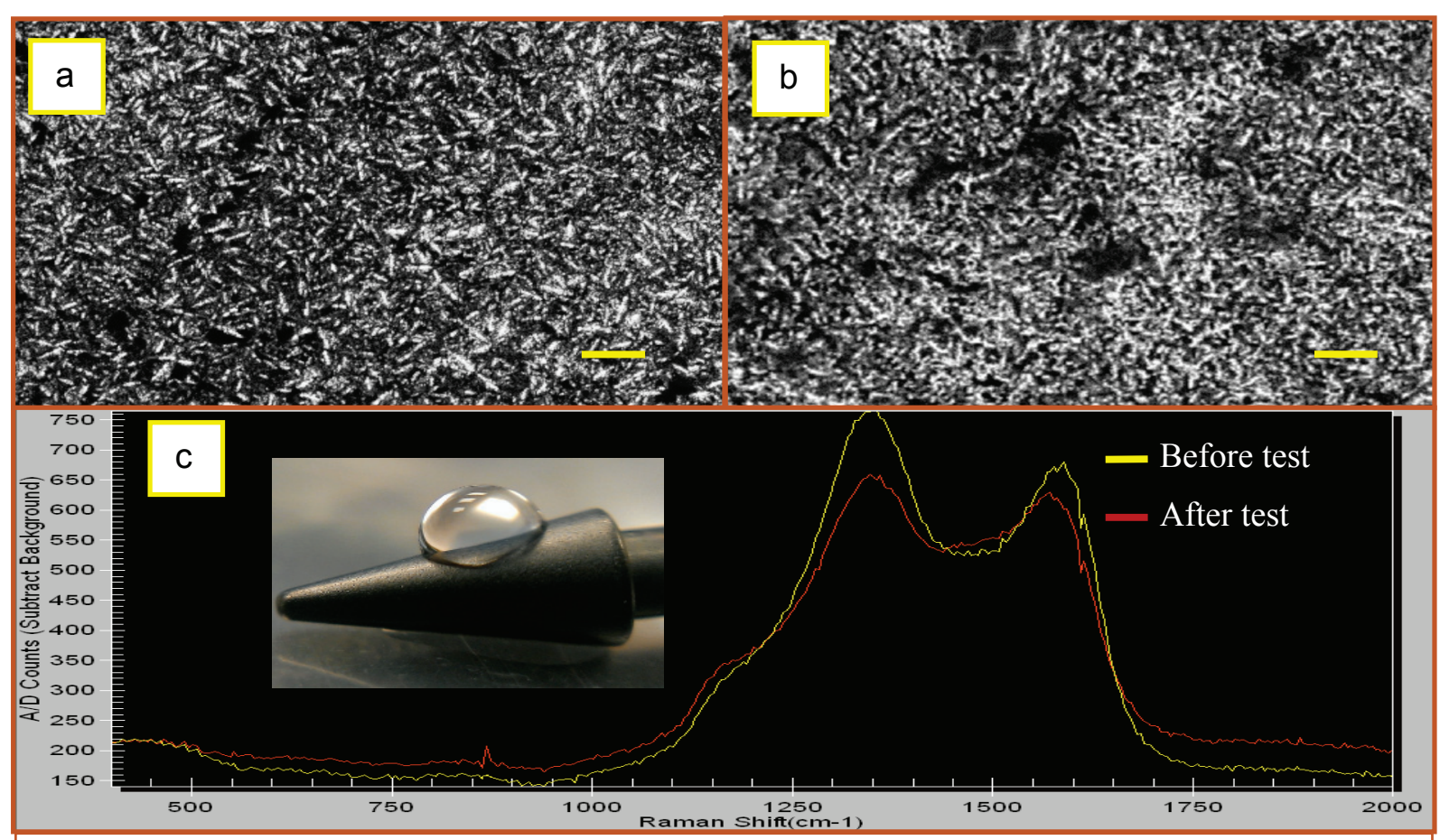

Fig.7 SEM image of UNCD film at a hard-hard contact area on a coated bearing a) before and b) after the plasma test. All scale bars represent $1 \mu \mathrm{m}$. c) Raman-spectrum comparison before and after the test. Insert of c) is a water drop sits on a tested bearing with a large contact angle.

\section{Acknowledgements}

The work was supported through a SBIR grant from NIH, R44HL108534. The Jarvik 2000 Heart components were provided by Jarvik Heart, Inc. SEM and AFM use of the Center for Nanoscale Materials (CNM), Argonne National Laboratory were supported by the U. S. Department of Energy, Office of Science, Office of Basic Energy Sciences, under Contract No. DE-AC02-06CH11357. The authors would like to thank Leonidas Ocola and Ralu Divan at CNM for their help on SEM and AFM imaging and thank Keith Gantz, Dale McClure and Jack Lusk for diamond film characterizations. 


\section{References}

[1] American Heart Association Statistical Update. Heart disease and stroke statisticsV2007 update. Circulation. 115:e69-e171, 2007.

[2] A. Grill, Diamond-like carbon coatings as biocompatible materials - an overview, Diamond and Related Materials 12, 166 (2003).

[3] V. L. Gott, D. E. Alejo, and D. E. Cameron, Mechanical Heart Valves: 50 Years of Evolution, Ann Thorac Surg. 76, S2230 (2003).

[4] I. R. McColl, et al, Low temperature plasma-assisted chemical vapour deposition of amorphous carbon films for biomedical-polymeric substrates, Diamond and related materials 3, 83 (1994)

[5] M. I. Jones, I. R. McColl, D. M. Grant, K. G. Parker, T. L. Parker, Protein adsorption and platelet attachment and activation, on TiN, TiC, and DLC coatings on titanium for cardiovascular applications, Journal of Biomedical Materials Research, 52(2), 413-421 (2000).

[6] Chinn, J.A., Horbett, T.A., Ratner, B.D. Baboon fibrinogen adsorption and platelet adhesion to polymeric materials," Thromb Haemost., 65(5):608-617 (1991).

[7] R. Hauert, C.V. Falub, G. Thorwarth, K. Thorwarth, Ch. Affolter, M. Stiefel, L.E. Podleska, G. Taeger, Retrospective lifetime estimation of failed and explanted diamond-like carbon coated hip joint balls, Acta Biomaterialia 8, 3170-3176 (2012).

[8] R. Hauert, G. Thorwarth, U. Müller, M. Stiefel, C.V. Falub, K. Thorwarth, T.J. Joyce, Analysis of the in-vivo failure of the adhesive interlayer for a DLC coated articulating metatarsophalangeal joint, Diamond \& Related Materials, 25, 34-39(2012).

[9] Bajaj,P., Akin, D., Gupta, A.R., Sherman, D., Shi, B., Auciello, O., Bashir, R. (2007) Ultrananocrystalline diamond film as an optimal cell interface for biomedical applications, Biomed Microdevices, 9:787-794. 
[10]Xiao, X., Wang J., Liu, C., Carlisle, J.A.,et. al,(2005) In vitro and in vivo evaluation of ultrananocrystalline diamond for coating of implantable retinal microchips”, J. Biomed.Mater Res. B., 77B(2): 273-281.

[11] Carlisle, J.A., and Auciello, O. (2003) Ultrananocrystalline Diamond: Properties and Applications in Biomedical Devices, The Electrochemical Society Interface, 12: 28-31.

[12] O. H. Frazier, Timothy J. Myers, Igor D. Gregoric, Tehreen Khan, Reynolds Delgado, Mihai Croitoru, Kathy Miller, Robert Jarvik, Stephen Westaby, Clinical Investigation and Reports: Initial Clinical Experience With the Jarvik 2000 Implantable Axial-Flow Left Ventricular Assist System, Circulation, 2002; 105: 2855-2860.

[13]Robert Jarvik, Blood pump bearings with separated contact surfaces, US Patent 7,762,941 B2, Jul.27, 2010.

[14] Hongjun Zeng, Wei Yin, Grace Catausan, Nicolaie Moldovan, John Carlisle, Ultrananocrystalline diamond integration with pyrolytic carbon components of mechanical heart valves, Diamond and Related Materials Volume 61, January 2016, Pages 97-101.

[15] Butler JE, Sumant AV. The CVD of nanodiamond materials. Chem Vap Deposition 2008;14:145-60.

[16] Liu H, Dandy DS. Studies on nucleation process in diamond CVD: an overview of recent developments. Diam Relat Mater 1995;4:1173-88.

[17] H. Zeng, A.R. Konicek, N. Moldovan, F. Mangolini, T. Jacobs, I.Wylie, P.U. Arumugam, S. Siddiqui, R.W. Carpick, J.A. Carlisle, Boron-doped ultrananocrystalline diamond synthesized with an H-rich/Ar-lean gas system, Carbon 84 (2015) 103.

[18] Ferrari AC, Robertson J. Origin of the 1150-cm_1 Raman mode in nanocrystalline diamond. Phys Rev B 2001;63. 121405-1-4.

[19] H. Zeng, P. U. Arumugam, and J. A. Carlisle, Fibrinogen adsorption study on ultrananocrystalline diamond as a biocompatible and antithrombogenic interfacial material for implantable devices, Phys. Status Solidi A 211(2014) 2785. 